\title{
58 THINKING ABOUT QUALITY IN QUALITATIVE INJURY PREVENTION RESEARCH-DOES HAVING DIFFERENT RESEARCHERS COLLECT DATA FOR THE SAME STUDY IMPACT ON THE QUALITY OF THE RESEARCH?
}

doi:10.1136/injuryprev-2012-040590w.58

\footnotetext{
1,2 J Stewart, ${ }^{1} \mathrm{D}$ Kendrick, ${ }^{1} \mathrm{P}$ Leighton. ${ }^{1}$ University of Nottingham, England; ${ }^{2}$ Nottinghamshire Healthcare, NHS Trust, England
}

Background In medical/health research it is increasingly recognised that whilst we may build evidence about what works using quantitative methods, we need to use qualitative methods to work out how best to implement it. Qualitative research is increasingly incorporated into injury research, which is frequently carried out by teams, working in different areas of the same country/different countries. However, evidence is emerging that different researchers, collecting data for the same qualitative, study can impact on the quality of the research.

Aim To explore the impact of different researchers collecting data for the same semi-structured qualitative interview research study being undertaken as part of a large multi-centre programme of injury prevention research in the UK.

Methods Semi-structured interviews undertaken with researchers working on the study explored what shaped the researchers approach to qualitative interviewing and the diversity of interview techniques. Framework analysis is ongoing. 
Results Data were collected from five researchers. Researchers had different perceptions of what was most important to explore in the interview, took different approaches to what they told participants about themselves whilst interviewing and to checking what the interviewee was saying. Further work includes exploring the outcome of the interviews undertaken by the different researchers assessing the impact on the data collected.

Contribution to the Field Qualitative research is an emerging method in injury prevention research. Using the example of current injury prevention research, this work offers insights into maintaining/ ensuring the quality of qualitative data collected by multiple researchers. 